MATEC Web of Conferences 22,03014 (2015)

DOI: $10.1051 /$ matec conf/ 20152203014

(C) Owned by the authors, published by EDP Sciences, 2015

\title{
Active Power Optimal Control of Wind Turbines with Doubly Fed In- ductive Generators Based on Model Predictive Control
}

\author{
Jiuwang Guo, Xingjie Liu \& Wang Wei \\ Electrical and Electronic Engineering Institute, North China Electric Power University, Baoding, Hebei, China
}

\begin{abstract}
Because of the randomness and fluctuation of wind energy, as well as the impact of strongly nonlinear characteristic of variable speed constant frequency (VSCF) wind power generation system with doubly fed induction generators (DFIG), traditional active power control strategies are difficult to achieve high precision control and the output power of wind turbines is more fluctuated. In order to improve the quality of output electric energy of doubly fed wind turbines, on the basis of analyzing the operating principles and dynamic characteristics of doubly fed wind turbines, this paper proposes a new active power optimal control method of doubly fed wind turbines based on predictive control theory. This method uses state space model of wind turbines, based on the prediction of the future state of wind turbines, moves horizon optimization, and meanwhile, gets the control signals of pitch angle and generator torque. Simulation results show that the proposed control strategies can guarantee the utilization efficiency for wind energy。 Simultaneously, they can improve operation stability of wind turbines and the quality of electric energy.
\end{abstract}

Keywords: doubly fed wind turbine; maximum wind energy tracking; state space model; predictive control

\section{INTRODUCTION}

In recent years, wind power technology has made rapid development and wind energy has become the main utilization form of renewable energy. In order to meet the development needs of the market, the unit capacity of wind turbines is getting in increasingly higher .For large wind turbines, the control system not only needs capture wind energy as much as possible to achieve the optimal power tracking, but also needs to achieve flexible operation of wind turbines, and thus it calls for higher requirements for the control system of wind turbines ${ }^{[1]}$

With the development of wind power technology, VSCF doubly fed wind turbines gradually become the current main model because of higher utilization rate for wind energy, adjustable power factors and other characteristics. When VSCF wind power system is running above, around and below the rated wind speed, it respectively has different control objectives and control strategies for each state. When it is running above the rated wind speed, variable pitch control technology is usually adopted to ensure the smooth output power; when it is running around the rated wind speed, maintaining a constant rotational speed is required to achieve the operation of constant rotational speed; when it is running below the rated wind speed, the rotational speed of wind wheel is required to vary with wind speed and maintain a constant optimal tip speed ratio to achieve the maximum wind energy capturing. Traditional active power control strategy of doubly fed wind turbines is the use of two independent PI controllers that respectively realize the control for the pitch angle of wind wheel and generator torque ${ }^{[2-4]}$. At present, the research on traditional control strategy has been relatively mature and also has gained extensive application in wind farms. Practice has proved that the traditional control strategy can better complete control tasks in wind farms. But with the higher requirement for wind power quality in power grids, under the traditional control strategy, power fluctuation of wind turbines and other issues also emerge.

This paper proposes a kind of active power predictive control method of doubly fed wind turbines within the range of full wind speed based on state space model of doubly fed wind turbines, and meanwhile, achieves the control for the pitch angle of wind wheel and generator torque. As a result of the adoption of state space model of wind turbines, we may fully take into account of the changes and constraints of each state variable in the control process, and thus different control objectives can be achieved by adjusting objective function at different wind speed segments. Predictive control has less demand for the accuracy of the model, strong robustness and other advantages, which may prevent excessive segmentation from making the models of wind turbines too complex, and meanwhile, may reduce the errors being brought by piecewise linearization of the models of wind turbines. The effectiveness of the proposed control strategy is verified through simulation analysis

\section{OPERATIONAL PRINCIPLES OF DOUBLY FED WIND TURBINES}

Doubly fed wind turbines comprise both energy conversion processes: from wind energy to mechanical 


\section{MATEC Web of Conferences}

energy and from mechanical energy to electrical energy. So wind turbines and generators are the main components for energy conversion. Wind turbines convert wind energy to mechanical energy; the generators convert mechanical energy to electrical energy The performance of wind wheel pitch angle and generator torque control system is directly related to the performance, energy conversion efficiency and power quality of the whole wind energy conversion system.

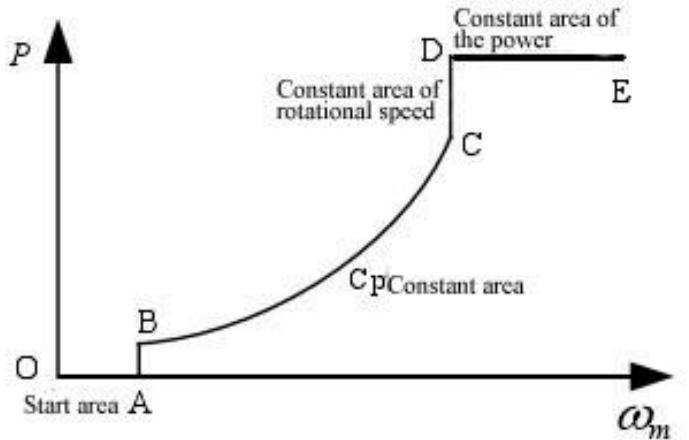

Figure 1. Operational area of wind turbines

According to the wind speed, the operational area of wind turbines may be divided into several kinds, as shown in Figure 1. When the wind speed is less than cut-in wind speed, the generators are separated from power grids so that wind turbines can't run to generate electricity. When wind speed reaches cut-in wind speed, rotor speed increases from zero to the speed that the generator can cut in, wind turbines started to be linked to power grids and run to generate electricity. On the basis of the power characteristics of wind turbines, wind turbines have the optimal running rotational speeds at different wind speeds, that is to say, at this time the capture efficiency for wind energy is the highest and the stress being applied to the wind turbines by the wind is the lowest. So the wind turbines are controlled to run at the optimized rotational speed, which can capture the maximum wind energy. Therefore, in the segment of low wind speed that is over cut-in wind speed, in order to fully take advantage of wind energy and improve operational efficiency of wind turbines, the resisting torque of the generators shall be adjusted to make the rotational speed vary with wind speed, so that the wind turbines are controlled to work in the area with the maximum utilization rate for wind energy; as the wind speed increases, the rotational speed of wind turbines is also increasing and ultimately reaches the maximum rotational speed allowed by wind turbines, which is also known as the rated rotational speed of wind turbines. Keeping the rotational speed constant, with the increasing of wind speed, the power of wind turbines increases, then the wind turbines work in constant area of rotational speed; as the power increases, the generators and the frequency changers ultimately reach the maximum power; as wind speed continues to increase, the power can be kept constant by increasing the pitch angle and reducing the absorption efficiency of wind wheels for the wind energy. At this time, the wind turbines work in constant area of the power ${ }^{[5]}$.

\section{STATE SPACE MODEL OF DOUBLY FED WIND TURBINES}

According to Betz theory, the wind energy being absorbed by wind wheel can be shown by expression (1)

$T_{t}=\frac{C_{p}(\lambda, \beta)}{\lambda} \frac{1}{2} \rho \pi R^{3} v^{2}$

Wherein: $T_{t}$ represents rotor torque; $R$ represents wind wheel radius; $C_{p}$ represents the utilization efficiency for wind energy; $\omega_{t}$ represents rotor speed; $\lambda$ represents tip speed ratio; $v$ represents wind speed; $\beta$ represents pitch angle.

In the case of keeping the pitch angle $\beta$ unchanged, there is an optimal tip speed ratio $\lambda_{\text {opt }}$ that makes the wind wheels have the highest utilization rate of wind energy, as shown in Figure 2.

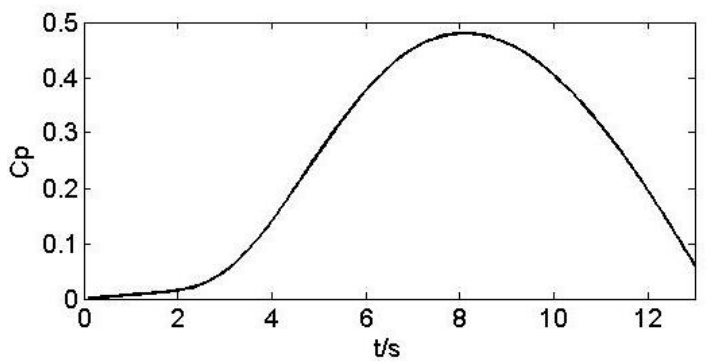

Figure 2. Characteristics curve of wind wheels absorbing wind energy

This wind wheel model has strong nonlinear characteristic, so it needs to be linearized to get the following:

$\delta T_{t}=L_{\omega}\left(\overline{\omega_{t}}, \bar{v}, \bar{\beta}\right) \delta \omega_{t}+L_{v}\left(\bar{\omega}_{t}, \bar{v}, \bar{\beta}\right) \delta v+L_{\beta}(\bar{\omega} t, \bar{v}, \bar{\beta}) \delta \beta(2)$

Wherein,

$L_{\omega}=\left.\frac{\partial T_{t}}{\partial \omega_{t}}\right|_{\left.\overline{(\omega}_{t}, \bar{v}, \bar{\beta}\right)}, \quad L_{v}=\left.\frac{\partial T_{t}}{\partial v}\right|_{\left(\bar{\omega}_{t}, \bar{v}, \bar{\beta}\right)}$,

$L_{\beta}=\left.\frac{\partial T_{t}}{\partial \beta}\right|_{\left(\bar{\omega}_{t}, \bar{v}, \bar{\beta}\right)}$

Wherein, $(\bar{\omega}, \bar{v}, \bar{\beta})$ is the point of reference; the values of $\bar{\omega}_{t}$ and $\bar{\beta}$ are decided by $\bar{v}$.

The mathematical models for transmission mechanism are as follows: 
$\frac{d \omega_{t}}{d t}=-\frac{N_{M}}{J_{t}} T_{t w}+\frac{1}{J_{t}} T_{t}$

$\frac{d \omega_{g}}{d t}=\frac{1}{J_{g}} T_{t w}-\frac{1}{J_{g}} T_{g}$

$\frac{d \omega_{g}}{d t}=\frac{1}{J_{g}} T_{t w}-\frac{1}{J_{g}} T_{g}+\frac{N_{M} B_{s}}{J_{t}} T_{t}+\frac{B_{s}}{J_{g}} T_{g}$

Wherein, $T_{t w}$ represents the torque of high speed spindle (HSS); $T_{g}$ represents generator torque; $\omega_{g}$ represents rotational speed of generators; $J_{t}$ and $J_{g}$ respectively represent rotational inertia of wind wheel and generators; $N_{M}$ represents the transformation ratio of transmission shaft; $k_{s}$ and $B_{s}$ respectively represent rigidity coefficient and damping coefficient.

The simplified models of generators are as follows:

$$
\frac{d T_{g}}{d t}=-\frac{1}{\tau_{g}} T_{g}+\frac{1}{\tau_{g}} T_{g}^{*}
$$

$P_{\mathrm{g}}=\eta T_{g} \omega_{g}$

Wherein, $\tau_{g}$ represents the equivalent time constant of generator torque adjustment; $\eta$ represents generator efficiency; $T_{g}^{*}$ represents setting value of the torque.

Mechanism model of pitch angle adjustment:

$\frac{d \beta}{d t}=-\frac{1}{\tau} \beta+\frac{1}{\tau} \beta^{*}$

Wherein, $\tau$ represents the equivalent time constant of pitch angle adjustment; $\beta^{*}$ represents setting value of pitch angle.

Take: $\quad x=\left[\delta \omega_{t} \delta \omega_{g} \delta T_{t w} \delta T_{g} \delta \beta\right] \quad, \quad u=$ $\left[\delta T_{g}^{*} \delta \beta^{*}\right], y=\left[\delta \omega_{g} \delta P\right]$ and achieve state space model of doubly fed wind turbines from expressions $1 \sim 8$ :

$$
\left\{\begin{array}{l}
\dot{x}(\mathrm{t})=\Phi x(\mathrm{t})+\Gamma_{u} u(\mathrm{t})+\Gamma_{v} \delta v(\mathrm{t}) \\
y=C x(\mathrm{t})
\end{array}\right.
$$

$$
\begin{aligned}
& \text { Wherein, } \\
& \Phi=\left[\begin{array}{ccccc}
\frac{L_{\omega}}{J_{t}} & 0 & -\frac{N_{M}}{J_{t}} & 0 & \frac{L_{\beta}}{J_{t}} \\
0 & 0 & \frac{1}{J_{g}} & -\frac{1}{J_{g}} & 0 \\
k_{s} N_{M}+\frac{N_{M} B_{s}}{J_{t}} L_{\omega} & -k_{s} & -\left(\frac{N_{M}^{2} B_{s}}{J_{t}}+\frac{B_{s}}{J_{g}}\right) & \frac{B_{s}}{J_{g}} & \frac{N_{M} B_{s}}{J_{t}} L_{\beta} \\
0 & 0 & 0 & -\frac{1}{\tau_{g}} & 0 \\
0 & 0 & 0 & 0 & \frac{1}{\tau}
\end{array}\right]
\end{aligned}
$$$$
\Gamma_{\mathrm{u}}=\left[\begin{array}{cc}
0 & 0 \\
0 & 0 \\
0 & 0 \\
\frac{1}{\tau_{g}} & 0 \\
0 & \frac{1}{\tau}
\end{array}\right] \quad \Gamma_{v}=\left[\begin{array}{c}
\frac{L_{v}}{J_{t}} \\
0 \\
\frac{N_{M} B_{s}}{J_{t}} L_{0} \\
0 \\
0
\end{array}\right]
$$

$C=\left[\begin{array}{ccccc}0 & 1 & 0 & 0 & 0 \\ 0 & \eta \bar{T}_{\mathrm{g}} & 0 & \eta \bar{\omega}_{\mathrm{g}} & 0\end{array}\right]$

\section{ACTIVE POWER PREDICTIVE CONTROL OF DOUBLY FED WIND TURBINES}

\subsection{Predictive control theory}

Predictive control is a kind of advanced control technology based on models, also known as model predictive control, and a new type of computer control algorithm that appeared in the mid and late 1970s in European and American industrial field. The present technology of model predictive control has been successfully applied in many fields such as petroleum, chemical, aerospace and energy.

The main features of predictive control are: on the basis of predictive model, use quadratic online rolling optimized performance index, feedback compensation and other control strategies to eliminate the modeling errors of the controlled objects, overcome the influence of structure, parameters, environment and other uncertain factors, and effectively make up for the problems on poor applicability of modern control theory facing complex controlled objects ${ }^{[6]}$. In consideration of the characteristics and running environment of doubly fed wind turbines, predictive control obviously has better applicability.

\subsection{Active power predictive control principles of doubly fed wind turbines}

The structure of active power control system of doubly fed wind turbines proposed in this paper is shown in Figure 3, which mainly consists of optimization function of control objective, predictive model and rolling optimization function. Optimization function of control objective determines the reference value $\omega \mathrm{g}^{*}$ of generator speed according to the current information of wind speed and in accordance with the principle of the maximum wind energy tracking; Pg* is set as rated power; MMPC controller selects the appropriate predictive model according to wind speed; Predictive controllers make predictions for the future state of the system based on the information feedback of current wind turbine state, and obtain a number of future control vectors by optimization calculation, in which $\mathrm{u}(\mathrm{k})$ value will be taken as the current actual control vector.

\subsection{Predictive Control}

Discretization is going to be made on the basis of this state space model to get predictive control model of wind turbines in each segment of wind speed:

$$
\begin{aligned}
& \left\{\begin{array}{l}
x^{i}(k+1)=A^{i} x^{i}(k)+B_{u}^{i} u(k)+B_{v}^{i} \delta v^{i}(k) \\
y^{i}(k)=C^{i} x^{i}(k)
\end{array}\right. \\
& \text { Wherein, } \\
& x^{i}(k)=\left[\begin{array}{lllll}
\delta \omega_{t}^{i}(k) & \delta \omega_{g}^{i}(k) & \delta T_{t w}^{i}(k) & \delta T_{g}^{i}(k) & \delta \beta^{i}(k)
\end{array}\right]
\end{aligned}
$$




\section{MATEC Web of Conferences}

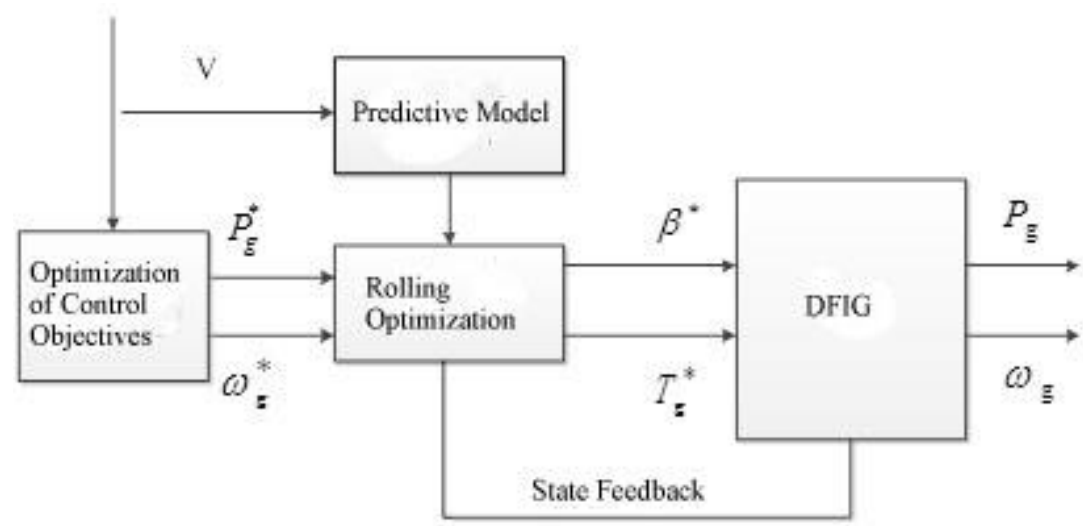

Figure 3. The structure of control system

$u(k)=\left[\delta T_{g}^{*}(k) \quad \delta \beta^{*}(k)\right]$

$y^{i}(k)=\left[\begin{array}{ll}\delta \omega_{g}^{i}(k) & \delta P_{g}^{i}(k)\end{array}\right]$

\subsection{Rolling optimization}

MMPC controller makes prediction for the future state according to expression (10) on the basis of feedback quantity of current wind turbine state and solves the following objective functions:

$$
\min J=\sum_{i=1}^{N_{p}}\left\|e^{i}(\mathrm{k})\right\|_{Q^{\prime}}^{2}+\sum_{j=0}^{N_{c}-1}\left(\|\Delta u(\mathrm{k}+j)\|_{R^{i}}^{2}+\|u(\mathrm{k}+\mathrm{j})\|_{R_{u}^{i}}^{2}\right)
$$

s.t

$$
\begin{aligned}
& \text { s.t } \quad \beta_{\min } \leq \Delta \beta^{*}(\mathrm{k}+\mathrm{j}) \leq \Delta \beta_{\max }, j=0,1, \ldots, N_{c}-1 \\
& \beta_{\min } \leq \beta^{*}(k+j) \leq \beta_{\max }, j=0,1, \ldots, N_{c}-1 \\
& 0_{\min } \leq T_{g}^{*}(k+j) \leq T_{\mathrm{g} \cdot \max }, j=0,1, \ldots, N_{c}-1 \\
& 0 \leq \omega_{g}^{i}(k+j) \leq \omega_{\mathrm{g} \cdot \max }, j=1,2, \ldots, N_{p} \\
& P_{g}^{i}(k+j) \leq P_{\mathrm{g} \cdot \max }, j=1,2, \ldots, N_{p}
\end{aligned}
$$

Wherein: $N_{p}$ and $N_{c}$ are respectively predictive time domain and control time domain,

$$
\begin{aligned}
& e^{i}(k)=\left[\omega_{g}^{*}(k)-\omega_{i}^{k}(k) \quad P_{\mathrm{g}}^{*}(k)-P_{\mathrm{g}}^{i}(k)\right]^{T}, \\
& Q^{i}=\operatorname{diag}\left(q_{1}^{i}, q_{2}^{i}\right), R^{i}=\operatorname{diag}\left(r_{1}^{i}, r_{2}^{i}\right), R_{u}^{i}=\operatorname{diag}\left(0, r_{3}^{i}\right)
\end{aligned}
$$

In different wind speed segments, the desired control effect can be achieved by selecting the appropriate weighting factor $q_{1}^{i}, q_{2}^{i}, r_{1}^{i}, r_{2}^{i}, r_{3}^{i}$. Specifically, in the segment of low wind speed, the main task of the controller is to adjust rotor speed to meet the optimal tip speed ratio to achieve maximum wind energy tracking At this stage, $r_{2}{ }^{i}$ and $r_{3}{ }^{i}$ take larger value to ensure that the pitch angle is maintained at 0 degree; $q_{2}{ }^{i}$ takes 0 ; $q_{l}^{i}$ and $r_{l}{ }^{i}$ select appropriate value to keep the output power of wind turbines smooth while tracking the maximum wind energy. When it is close to rated wind speed, the values of $r_{2}{ }^{i}$ and $r_{3}{ }^{i}$ need to be reduced, and meanwhile, $q_{2}{ }^{i}$ takes lower value and the pitch angle is allowed to be adjusted appropriately to prevent the output power from exceeding the rated value. In the segment of high wind speed, generator speed and output power are made to stabilize at the rated value primarily by adjusting the pitch angle. At this stage, the value of $q_{2}{ }^{i}$ needs to be increased, and meanwhile, $r_{2}{ }^{i}$ and $r_{3}{ }^{i}$ take lower value to bring greater degree of freedom to the adjustment of pitch angle.

\section{SIMULATION ANALYSIS}

Table 1: Main parameters of $1.5 \mathrm{MW}$ doubly fed wind turbines

\begin{tabular}{l|l}
\hline rated power & $1.5 \mathrm{MW}(0.9$ p.u. $)$ \\
\hline rated speed of wind wheel & $23 \mathrm{rpm}(1.2$ p.u. $)$ \\
\hline $\max /$ min speed of wind wheel & $9.5 / 25 \mathrm{rpm}$ \\
\hline wind wheel radius & $35 \mathrm{~m}$ \\
\hline rated wind speed & $11 \mathrm{~m} / \mathrm{s}$ \\
\hline cut-in/cut-out wind speed & $4 / 26 \mathrm{~m} / \mathrm{s}$ \\
\hline transformation ratio of transmission shaft & 62.6 \\
\hline inertia time constant of wind wheel & $6 \mathrm{~s}$ \\
\hline inertia time constant of generators & $1 \mathrm{~s}$ \\
\hline$\beta_{\min } / \beta_{\max }$ & $0 / 45^{\circ}$ \\
$\dot{\beta}_{\min } / \dot{\beta}_{\max }$ & $-10 / 10^{\circ} / \mathrm{s}$ \\
\hline
\end{tabular}

In order to verify the feasibility of the proposed control strategy, a simulation study on a $1.5 \mathrm{MW}$ doubly fed wind turbine is going be carried out and a comparison with the control effect of conventional PI con- 
troller will be made.

Main parameters of doubly fed wind turbines are shown in Table 1:

Figures 2-4 are the comparison of control effects of both control strategies that are respectively in the segment of low wind speed, around rated wind speed, and in the segment of high wind speed:

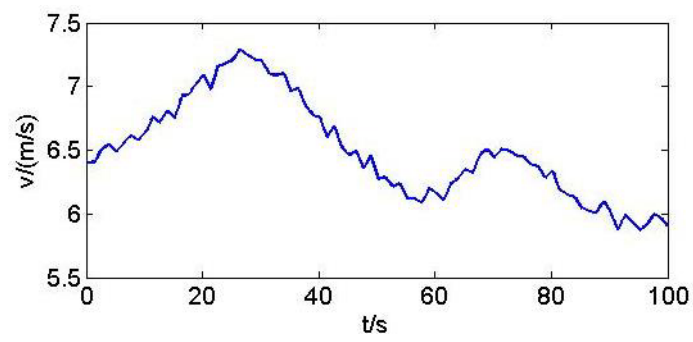

a

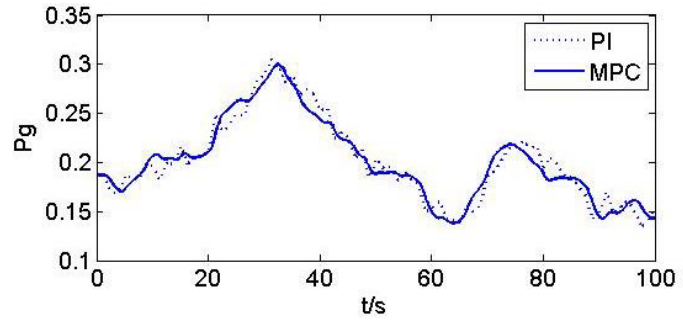

b

Figure 2. Simulation results in the segment of low wind speed

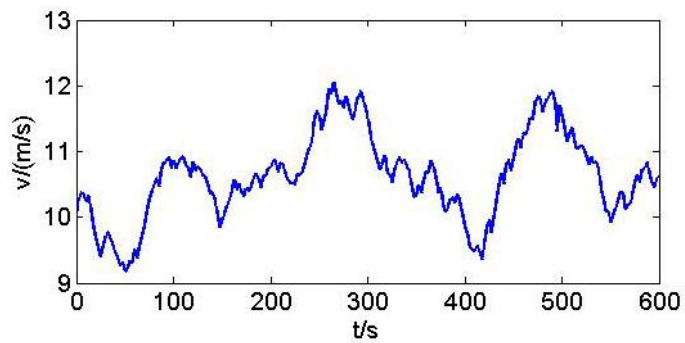

a

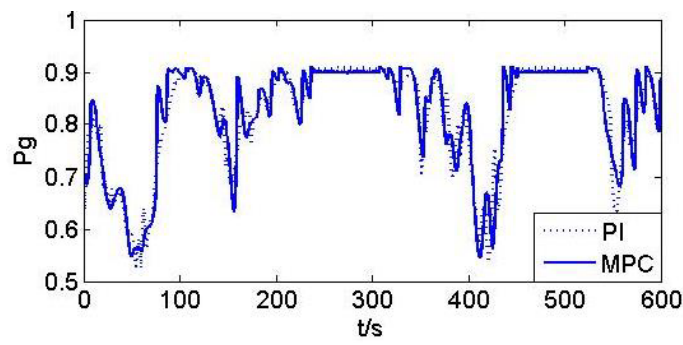

b

Figure 3. Simulation results around rated wind speed
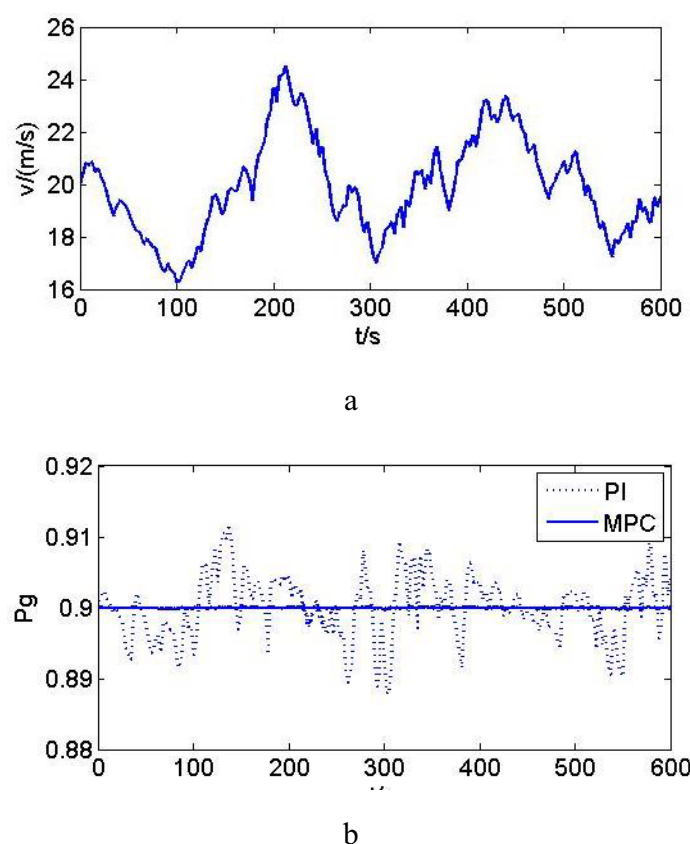

Figure 4. Simulation results in the segment of high wind speed

Through the simulation results, we may see that both kinds of control strategies can vary with the wind speed and control wind turbines to track maximum wind energy or keep the wind turbines fully running. But compared with the traditional control strategies, the control strategy mentioned herein has better control effect in reducing the fluctuation of the output power and the guarantee of output power quality. In the segment of low wind speed, MPC and PI controllers can make the generator speed achieve optimal speed to reach the purpose of tracking maximal wind energy. Although there are some fluctuations on their output power, compared with conventional PI control, the fluctuation of the output power under MPC control strategy has been reduced slightly, which is because MPC not only considers current wind speed and wind turbine state but also makes predictions for wind turbine state according to some future wind speed; on the basis of comprehensive consideration, the current control vector is produced, which may reduce the influence caused by random fluctuation of wind speed. Around rated wind speed, the output power overshoot under MPC control strategy has been significantly reduced and the transition from maximum wind energy tracking to full-load operation has been realized more stably. In the segment of high wind speed, MPC makes the fluctuation of output power reduced greatly and makes the output power stabilized around the rated value, which fully embodies the superiority of the control strategy proposed in this paper. 


\section{MATEC Web of Conferences}

\section{SUMMARY}

A kind of multi-model active power predictive control strategy of doubly fed wind turbines based on state space model has been proposed against the problems in the existing active power control system of doubly fed wind turbines, which achieves the control for the output power of wind turbines by simultaneously controlling the generator torque and the pitch angle of wind wheel. Simulation results show that the control effect of the proposed control strategy has been obviously improved compared with the existing PI control strategy. In the segment of low wind speed, $M M P C$ controller may significantly reduce the fluctuation of output power while tracking the maximum wind energy; around rated wind speed, $M M P C$ controller can reduce the overshoot of the output power to achieve the smooth transition from the maximum wind energy tracking to full load power generation. In the segment of high wind speed, improvement effect of $M P P C$ controller is particularly evident; the fluctuation of the generator speed has been reduced significantly; the wind turbines run smoothly under rated state.

\section{ACKNOWLEDGEMENTS}

This work was supported by the Natural Science Foundation of Hebei Province of China (No.E2015502066).

\section{REFERENCES}

[1] He, Y.1., Huang, S., Su, D.X., Zhou, H.L., Jin, X. \& Wang, L. 2012. Maximum Wind Energy Tracking of Variable Speed Wind Turbines and Blade Pitch Control. Control Engineering, 03: 523-526 + 538 .

[2] Lei, T. \& Jia, L.M. 2011. Nonlinear Predictive Control Algorithm of Variable Pitch of Wind Turbines. Computer Simulation, 01: 319-322 + 335 .

[3] Xia, C.L. \& Song, Z.F. 2007. Variable Pitch ADRC of VSCF Wind Power Generation System. Proceedings of the CSEE, 27 (14): 91-95.

[4] Zhao, Y.X., Xia, C.L. \& Song, Z.F., etc. 2008. Nonlinear PID Control of Wind Turbine Speed of VSCF Wind Power Generation System. Proceedings of the CSEE, 28 (11): 133-138

[5] Zhao, Y. 2008. A Study On Vector Control Technology of Doubly Fed Induction Generators Used In Wind Power Generation System, Huazhong University of Science and Technology.

[6] Jing, Y.J. 2011. A Study on Model Predictive Control of Doubly Fed Wind Turbines, Shenyang University of Technology.

[7] Wei, S.B. 2009. A Study on Distributed Predictive Control Method in Multi-Agent System, Chongqing University

[8] Henriksen, L.C., Hansen, M.H. \& Poulsen, N.K. 2012. Wind turbine control with constraint handling: a model predictive control approach. Control Theory \& Applications, IET, vol.6, no.11, July 19, pp: 1722, 1734.

[9] Soliman, M., Malik, O.P. \& Westwick, D.T. 2011. Multiple Model Predictive Control for Wind Turbines with Doubly Fed Induction Generators. Sustainable Energy, IEEE Transactions on, vol.2, no.3, July, pp: 215, 225. 